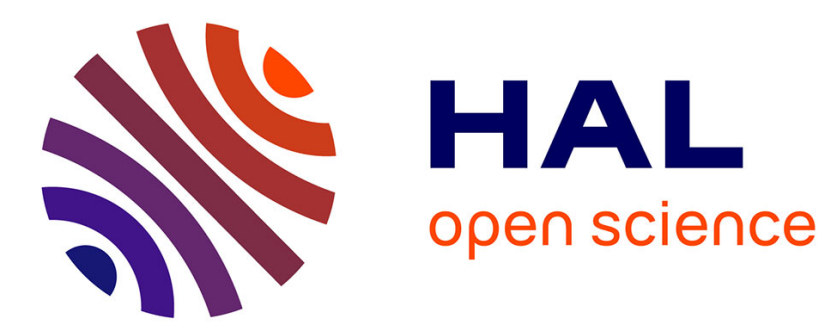

\title{
On stratification control of the velocity fluctuations in sedimentation
}

\author{
Daniel Chehata Gomez, Laurence Bergougnoux, E. John Hinch, Elisabeth \\ Guazzelli
}

\section{> To cite this version:}

Daniel Chehata Gomez, Laurence Bergougnoux, E. John Hinch, Elisabeth Guazzelli. On stratification control of the velocity fluctuations in sedimentation. Physics of Fluids, 2007, 19, pp.098102. 10.1063/1.2772902 . hal-01067004

\section{HAL Id: hal-01067004 https://hal.science/hal-01067004}

Submitted on 22 Sep 2014

HAL is a multi-disciplinary open access archive for the deposit and dissemination of scientific research documents, whether they are published or not. The documents may come from teaching and research institutions in France or abroad, or from public or private research centers.
L'archive ouverte pluridisciplinaire HAL, est destinée au dépôt et à la diffusion de documents scientifiques de niveau recherche, publiés ou non, émanant des établissements d'enseignement et de recherche français ou étrangers, des laboratoires publics ou privés. 


\title{
On stratification control of the velocity fluctuations in sedimentation
}

\author{
Daniel Chehata Gómez and Laurence Bergougnoux \\ IUSTI-CNRS UMR 6595, Polytech'Marseille, Technopôle de Château-Gombert, \\ 13453 Marseille Cedex 13, France \\ John Hinch \\ DAMTP, University of Cambridge, Wilberforce Road, Cambridge CB3 OWA, United Kingdom \\ Élisabeth Guazzelli \\ IUSTI-CNRS UMR 6595, Polytech'Marseille, Technopôle de Château-Gombert, \\ 13453 Marseille Cedex 13, France
}

(Received 21 May 2007; accepted 27 July 2007; published online 11 September 2007)

\begin{abstract}
We have tested whether stratification can govern local velocity fluctuations in suspensions of sedimenting spheres. Comparison of the proposed scaling for local control of fluctuations by stratification to experimental data demonstrates that this mechanism cannot account for the reduction of the observed velocity fluctuations. (ㅇ 2007 American Institute of Physics.
\end{abstract}

[DOI: $10.1063 / 1.2772902]$

Although the sedimentation of non-Brownian particles at low Reynolds number can be considered as one of the simplest examples of suspension flow, it is still a challenging problem, still debated today. The difficulty lies in the long range nature of the multibody hydrodynamic interactions between particles and the coupling between these and the microstructure of the suspension.

A single rigid sphere settles at the Stokes velocity $V_{S}=(2 / 9) a^{2}\left(\rho_{p}-\rho_{f}\right) g / \mu$, where $a$ is the sphere radius, $\rho_{p}$ the density of the particles, $\rho_{f}$ that of the fluid, and $\mu$ the fluid viscosity. A uniform suspension of spheres in a vessel settles more slowly than a single sphere. The main hindrance effect comes from the fluid back-flow induced by the particles settling towards the impenetrable bottom of the settling vessel. At very low volume fractions, the theoretical problem has been solved by Batchelor, ${ }^{1}$ who found the first correction in concentration $\phi$ to the Stokes velocity assuming uniformly dispersed spheres. At larger volume fractions, only sophisticated computer-simulation can capture the phenomenon.

The mean velocity does not completely characterize the sedimentation process. Individual particle motions fluctuate about the mean as the suspension microstructure constantly changes during the settling process. These fluctuations are observed experimentally to be very large and anisotropic, ${ }^{2}$ but still defy theoretical predictions. The standard theoretical argument developed by Caflisch and Luke ${ }^{3}$ and Hinch $^{4}$ assumes that the random initial mixing of the suspension creates statistical fluctuations in particle number $\sqrt{N}$ (Poisson statistics) in "blobs" of all length scales $l$ from the container size down to mean interparticle spacing $a \phi^{-1 / 3}$. Balancing the fluctuations in the weight $(4 / 3) \pi a^{3} \sqrt{ } N\left(\rho_{p}-\rho_{f}\right) g$ against a Stokes drag on the blob $6 \pi \mu l V_{c}$ yields convection currents, also called "swirls," of amplitude $V_{c}=V_{S} \sqrt{\phi l / a}$. The dominant fluctuations are set by the smallest cell dimension; i.e., the fluctuation magnitude scales with the depth of the container $D$ (the cell is of size $H \times W \times D$, where $H$ is the cell height along gravity and $W \times D$ the cell section with $D$ being the smallest dimension). Therefore, they diverge with the size of the container.

This divergence is not seen (at least to persist) in most experiments. ${ }^{5-7}$ Experiments in large containers ${ }^{7,8}$ show that large-scale fluctuations (of size $\approx$ cell width $W$ ) indeed dominate the dynamics just after the initial mixing of the suspension. However, they are transient and smaller-scale fluctuations (of size $\approx 20 a \phi^{-1 / 3}$ ) remain and prevail until the arrival of the upper sedimentation front. The scaling of the size of these small-scale fluctuations is still an open question.

The idea that stratification may suppress the divergence of the velocity fluctuations was first suggested by Bławdziewicz around 1995, but remained unpublished. It was only later in 2000 that the argument was proposed by Luke, ${ }^{9}$ who showed that stratification can lead to fluctuation decay. Tee et al. ${ }^{10}$ and Mucha and Brenner ${ }^{11}$ proposed a theoretical model based on this idea. Their main argument is that a very small vertical stratification caused in particular by the broadening of the sedimenting front can cause the fluctuations to decrease below those of an independent uniform distribution of particles.

The scaling argument showing that stratification can inhibit the velocity fluctuations goes as follows. The size of the fluctuations discussed above is limited when the statistical fluctuations in volume fraction $\sqrt{\phi\left(a^{3} / l^{3}\right)}$ are comparable to the variation in volume fraction due to the stratification $-l d \phi / d z$, where $-d \phi / d z$ is the concentration gradient in the direction of gravity. This gives a cutoff length of scale $a \phi^{1 / 5}(-a d \phi / d z)^{-2 / 5}$, yielding the largest remaining fluctuations of magnitude $V_{S} \phi^{3 / 5}(-a d \phi / d z)^{-1 / 5}$. This argument for control of fluctuations by stratification only applies when the cutoff length is smaller than the cell depth $D$. There is therefore a critical stratification when the cutoff scale is equal to $D$; i.e., $(-a d \phi / d z)_{c} \sim(a / D)^{5 / 2} \phi^{1 / 2}$. When $-a d \phi / d z>(-a d \phi / d z)_{c}$, stratification should govern the fluctuations. Note this argument requires $-d \phi / d z$ to be constant over the cutoff length scale.

The objective of the present Brief Communication is to 
TABLE I. Particle characteristics of the two batches: mean radius $\langle a\rangle$, standard deviation $\sigma_{a}$, particle density $\rho_{p}$, and Stokes velocity $V_{S}=(2 / 9)$ $\times\langle a\rangle^{2}\left(\rho_{p}-\rho_{f}\right) g / \mu$.

\begin{tabular}{ccccc} 
Batch & $\langle a\rangle(\mathrm{cm})$ & $\frac{\sigma_{a}}{\langle a\rangle}(\%)$ & $\rho_{p}\left(\mathrm{~g} / \mathrm{cm}^{3}\right)$ & $V_{S}(\mathrm{~cm} / \mathrm{s})$ \\
\hline A & 0.0148 & 5.6 & $4.11 \pm 0.07$ & $0.0150 \pm 0.0018$ \\
B & 0.0152 & 2.5 & $4.11 \pm 0.07$ & $0.0158 \pm 0.0010$ \\
\hline \hline
\end{tabular}

examine experimentally whether stratification can control velocity fluctuations in suspensions of sedimenting spheres. Particle velocity fluctuations are measured during the sedimentation process in an imaging window covering $\approx 1 / 4$ of the cell height $H(\approx$ the size of the largest swirls, which are of the size of the cell width: $W=D=H / 4)$. The predicted fluctuations are inferred from average concentration and vertical concentration gradient also measured within the same imaging window, as the theory is local to a scale large relative to the scale of the swirls. The proposed scaling can then be compared to the measured fluctuations.

The sedimentation experiments were performed in a glass-walled vessel of cross section $10 \times 10 \mathrm{~cm}^{2}[\approx 100$ $\left.\times 100\left(a \phi_{0}^{-1 / 3}\right)^{2}\right]$ filled with a fluid height $H=40 \mathrm{~cm}$ $\left(\approx 400 a \phi_{0}^{-1 / 3}\right)$. Two batches of glass spheres having slightly different polydispersities were used to perform experiments with the same initial volume fraction $\phi_{0}=0.3 \%$ (see Table I). The suspending fluid was silicone oil 47V1000, which had a viscosity $\mu=10.0 \pm 0.3 \mathrm{P}$ and a density $\rho_{f}=0.965 \pm 0.007 \mathrm{~g} / \mathrm{cm}^{3}$ at the air-conditioned room temperature of $25 \pm 1{ }^{\circ} \mathrm{C}$. For these combinations of particles and fluid, the particle Reynolds number is $\langle a\rangle V_{S} \rho_{f} / \mu \approx 2 \times 10^{-5}$, and the Péclet number is large.

Each sedimentation experiment initially consisted of mixing the suspension by moving a small propeller (of size $4 \mathrm{~cm}$ and speed $2000 \mathrm{rpm}$ ) within the suspension for $\approx 10 \mathrm{~min}$ in order to obtain a visually uniform particle distribution throughout the suspension. The difficulty lies in making a homogeneous mixing for each run. In order to obtain reproducible results, the mixing was always performed in the same way for the different runs. First, the sediment was turned into a concentrated homogeneous suspension in the bottom $5 \mathrm{~cm}$ of the vessel. Secondly, the mixing was extended to a volume twice as high. This procedure of doubling the mixed volume was repeated until the complete volume of the cell was homogenized. The starting time of each experimental run corresponded to the cessation of mixing. Other types of mixing were attempted, but only the above protocol was successful in producing a visually uniform mixing over the suspension height.

First, particles velocities were measured using particle image velocimetry (PIV). A thin light-sheet (of thickness $\approx 1 \mathrm{~mm}$ ) produced by two $15 \mathrm{~mW}$ laser diodes facing each other was employed to illuminate the median plane of the cell. A $(1040 \times 1392$ pixel $)$ charge coupled device digital camera (Basler A102f) placed at right angles to the lightsheet was focused on the illuminated particles which scattered the light. It sampled the entire cell cross section for a window of height $10 \mathrm{~cm}$ placed $25.5 \mathrm{~cm}$ below the liquid-air interface. Pairs of images separated by approximately one Stokes time $=a / V_{S}$ were captured every $40 \mathrm{~s}$ (with IMAGEJ available at http://rsb.info.nih.gov/ij/) during the sedimentation process. Using a PIV code developed under MATLAB, the pairs were processed to obtain a two-dimensional velocityvector map. In practice, this involved discretizing each image into a map of $33 \times 44$ nodes. In a small interrogation region explored around each node, the local particle displacement between the two images was then computed by cross-correlation. The spatial resolution of the measurement was determined by the size of the interrogation region, which was $64 \times 64$ pixels $^{2}\left[\approx 0.5 \times 0.5 \mathrm{~cm}^{2} \approx 5 \times 5\left(a \phi_{0}^{-1 / 3}\right)^{2}\right]$ in order to contain enough particles to obtain reliable measurements. At each captured time, we computed the vertical mean velocity and standard deviation $V_{\text {exp }}^{\prime}$ from all velocity data coming typically from ten experimental runs.

Secondly, the light attenuation produced by the sedimenting suspension was measured within the same imaging window with the same camera and digital imaging system, but with the cell being back-lit by two neon tubes. ${ }^{8}$ To begin with, we determined the calibration law giving the light attenuation as a function of the mean particle volume fraction $\phi_{0}$. Using this calibration law, the average concentration $\phi$ within the imaging window was computed by averaging the gray-scale intensities over the entire image. By summing the intensities over the horizontal lines of the image, the vertical intensity profile was also determined. A linear fit of this profile provided the vertical gradient $-d \phi / d z$. Using these measurements of the average concentration and concentration gradient, we calculated the predicted vertical velocity fluctuations as $V_{\mathrm{th}}^{\prime} / V_{S}=k \phi^{3 / 5}(-a d \phi / d z)^{-1 / 5}$, with $k$ being the only adjustable parameter.

Figure 1 (top) shows the average concentration $\phi$ and gradient $-a d \phi / d z$ in the imaging window for a suspension of particles of batch A (polydispersity of 5.6\%). Three measurements $(\bigcirc, \square, \triangle)$ coming from three different sedimentation runs are displayed. There is a good reproducibility of the initial mixing process and of the subsequent evolution of $\phi$ and $-a d \phi / d z$. The average concentration $\phi$ presents a very slow decrease until $t / t_{S} \approx 1000$ and then drops as the sedimentation front crosses the imaging window. The initial gradient $-a d \phi / d z$ created by the mixing of the suspension is consistently $\approx 10^{-7}$, and remains approximately constant for $t / t_{S} \lesssim 1000$. Beyond that time, the front arrival results in a strong increase of the vertical concentration gradient, which reaches a maximum $\approx 3.3 \times 10^{-6}$ at $t / t_{S} \approx 1800$ and then decreases. The error bar in $\phi$ is $\approx 10^{-5}$, and that in $-a d \phi / d z$ is $\approx 10^{-13}$. Note that the measured concentration gradient is always larger than the critical gradient $\lesssim 10^{-9}$.

Figure 1 (bottom) juxtaposes the time evolution of $V_{\text {th }}^{\prime}$ and $V_{\text {exp }}^{\prime}$. The velocity fluctuations $V_{\exp }^{\prime}$ obtained from PIV measurements are initially large $\left(\approx 1.3 V_{S}\right)$. They undergo a strong decrease to a uniform value $\approx 0.5 V_{S}$ at $t / t_{S} \approx 300$. A much smaller decrease is experienced for $t / t_{S} \gtrsim 1000$, when the sedimentation front reaches the imaging window. The typical error bars in $V_{\text {exp }}^{\prime}$ are computed with the usual propagation of errors (statistical error in velocity fluctuations and experimental error in Stokes velocity). The predicted $V_{\text {th }}^{\prime}$ coming from the different runs $(\bigcirc, \square, \triangle)$ remains constant 

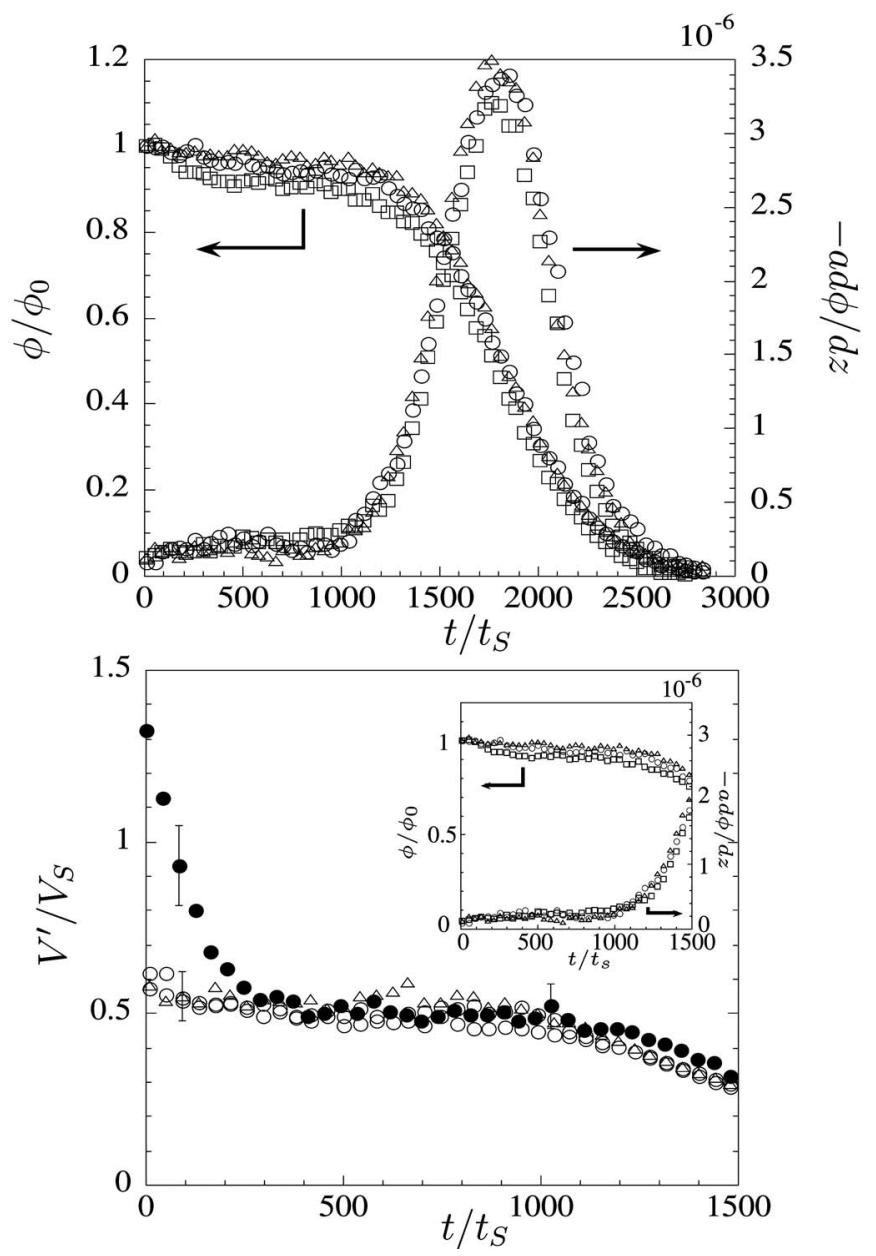

FIG. 1. Average concentration $\phi$ and gradient $-a d \phi / d z$ versus time (top and bottom inset) and comparison of the time evolution of $V_{\mathrm{th}}^{\prime}(\bigcirc, \square, \triangle)$ and $V_{\text {exp }}^{\prime}(\bullet)$ (bottom) for particles of batch A.

until $t / t_{S} \approx 1000$ and after that time presents a small decrease. The parameter $k$ has been fixed at 0.8 to match the uniform experimental value of 0.5 and the error bars have been computed with the usual propagation of errors. Clearly, while the proposed scaling fails in predicting the initial strong decrease of the velocity fluctuations, it succeeds in reproducing the adjusted constant value and the small decrease due to the front arrival into the imaging window. Note, however, that it is not a very convincing verification of the proposed scaling (with weak power law) as both the gradient and concentration do not present large variation in the explored time-range (see bottom inset of Fig. 1).

Figure 2 (top) also displays the average concentration $\phi$ and gradient $-a d \phi / d z$, but for a suspension of particles of batch B (polydispersity of 2.5\%). Three measurements $(O$, $\square, \triangle$ ) coming from three different runs are again presented and indicate the good reproducibility of the experiments. The average concentration $\phi$ shows a small decline until $t / t_{S} \approx 1000$. Beyond this time, $\phi$ rapidly decreases due to the influence of the sedimentation front. The initial gradient $-a d \phi / d z$ created by the mixing of the suspension is systematically slightly negative $\left(\approx-10^{-7}\right)$ in the imaging window up to $t / t_{S} \approx 1000$. The front arrival at $t / t_{S} \approx 1000$ leads again to a strong increase of $-a d \phi / d z$ followed by a decrease after

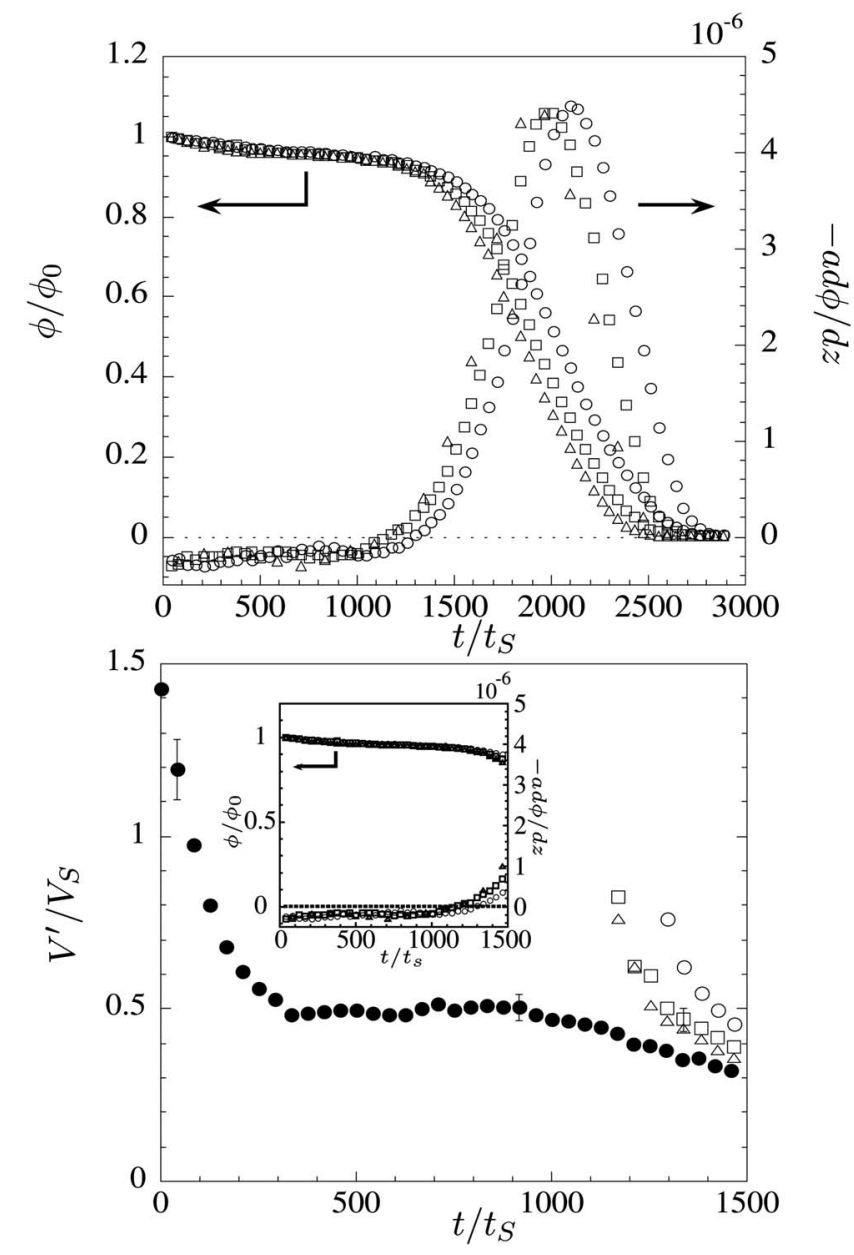

FIG. 2. As for Fig. 1, but for particles of batch B.

a maximum $\approx 4.5 \times 10^{-6}$ reached at $t / t_{S} \approx 2000$. The fact that the initial gradient is negative while the concentration decreases seems inconsistent at first sight. In fact, the gradient is slightly positive above the present imaging window.

Figure 2 (bottom) compares the time evolution of $V_{\text {th }}^{\prime}$ and $V_{\text {exp }}^{\prime}$. The time evolution of $V_{\exp }^{\prime}$ obtained from PIV measurements is identical to that of $V_{\exp }^{\prime}$ in Fig. 1 within error tolerance (see also Fig. 3). The predicted fluctuations $V_{\mathrm{th}}^{\prime}$ can only be computed for positive values of the vertical gradient $-a d \phi / d z$ as the scaling contains a negative power law. We have thus plotted $V_{\text {th }}^{\prime}$ coming from the different runs $(\bigcirc, \square$, $\triangle$ ) when this gradient is positive, keeping the adjustable parameter at the same value $k=0.8$. Only the small decrease due to the front arrival is poorly reproduced.

Figures 1 and 2 show a very robust evolution of $V_{\exp }^{\prime}$ with an initial strong decrease followed by a constant value $\approx 0.5 V_{S}$, whatever initial gradient is created by the mixing of the suspension. Interestingly, a fixed mixing procedure seems to have interacted with the particles of different polydispersity to produce either a positive (stably stratified) or negative (unstably stratified) initial gradient for more or less polydisperse particles, respectively. The proposed prediction can only roughly reproduce the decrease of the velocity fluctuations within the sedimentation front. This decrease might be explained by the effect of polydispersity (even small) of the 


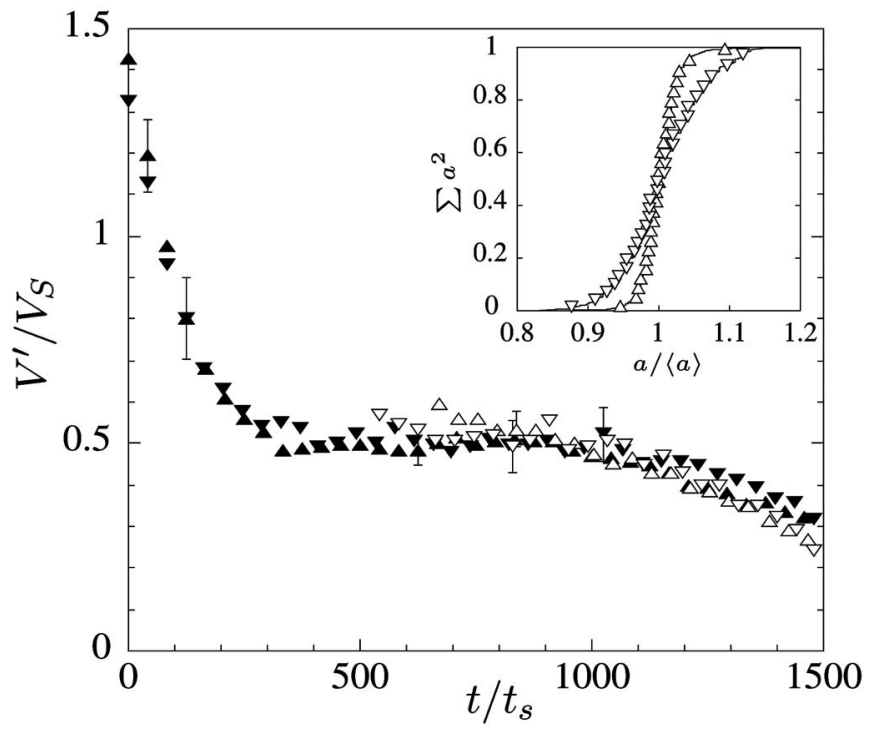

FIG. 3. Comparison of the time evolution of $V_{\text {poly }}^{\prime}(\nabla$ for particles $A$ and $\triangle$ for particles B) and $V_{\exp }^{\prime}(\boldsymbol{\nabla}$ for particles $\mathrm{A}$ and $\boldsymbol{\Delta}$ for particles B) and normalized cumulative sum of the square of the radius, $a^{2}$, versus dimensionless radius, $a /\langle a\rangle$ for the two batches of particles $\mathrm{A}(\nabla)$ and $\mathrm{B}(\triangle)$ (inset).

particles as the front is a special region depleted of the larger heavier particles. Of course, the magnitude of the fluctuations cannot be explained by the sole effect of polydispersity $\left(\approx 2 \sigma_{a} /\langle a\rangle\right)$. A theory combining the effects of polydispersity and hydrodynamic interaction is lacking, but we can try a very crude estimation using an adjustable coefficient. From the measured particle-radius distribution, we can compute the cumulated distributions of the square of the radius (see Fig. 3, inset). Considering that the light attenuation technique probes the surfaces of the particles, we can relate, at each time, the reduction percentage in $\phi / \phi_{0}$ [see Figs. 1 and 2 (top)] to the corresponding percentage in the cumulated distribution (see Fig. 3, inset) and infer the largest radius $a_{\phi / \phi_{0}}\left(t / t_{S}\right)$ found in the imaging window versus time. We can then estimate the time evolution of the fluctuations as $V_{\text {poly }}^{\prime} / V_{S}=k^{\prime}\left[a_{\phi / \phi_{0}}^{2}\left(t / t_{S}\right)-\langle a\rangle^{2}\right] /\langle a\rangle^{2}$. This crude polydispersity model reproduces the observed decrease in the front as seen in Fig. 3 with the adjustable coefficient being $k^{\prime}=2.6$ and 4.6 for particles of batches A and B, respectively. The estimated $V_{\text {poly }}^{\prime}$ is an average coming from the three concentration measurements and the error tolerance corresponds to the standard deviation. Lastly, note that the mean vertical velocity (not represented in figures) stays $\approx V_{S}$ outside the front as hindered settling plays no role in these very dilute suspensions and also presents a decrease as the front reaches the imaging window, as already reported by Bergougnoux et $a l^{8}$

In conclusion, we have compared experimental velocity fluctuations measured by PIV technique to theoretical predictions deduced from average concentration and concentration gradient measured by light attenuation method. Clearly, stratification does not control the reduction of the velocity fluctuations. The decay of the large-scale fluctuations giving way to smaller-scale fluctuations seems to be a very robust feature that is insensitive to the initial gradient created by the mixing. This finding seems to support the idea that the largescale convection currents created by the mixing are transient (as the heavy blobs settle to the bottom and the light blobs rise to the top) and eventually leave behind smaller-scale fluctuations. ${ }^{4}$ The stratification theory relies on the strong hypothesis that particles are randomly distributed for all length scales; i.e., Poisson statistics for all length scales. This assumption is debatable as the mixing of the suspension may not lead to a random distribution of particles in this nonequilibrium problem of statistical mechanics. The outcome of the experimental mixing may not be the random positioning of the particles of the theory but something yet to be quantified.

We wish to thank A. Daerr for his help in developing some IMAGEJ macros and P. Meunier and T. Leweke for providing the PIV code. Support from CONACyT is gratefully acknowledged by D.C.G.

${ }^{1}$ G. K. Batchelor, "Sedimentation in a dilute dispersion of spheres," J. Fluid Mech. 52, 245 (1972).

${ }^{2}$ H. Nicolai, B. Herzhaft, E. J. Hinch, L. Oger, E. Guazzelli, "Particle velocity fluctuations and hydrodynamic self-diffusion of sedimenting nonBrownian spheres," Phys. Fluids 7, 12 (1995).

${ }^{3}$ R. E. Caflisch and J. H. C. Luke, "Variance in the sedimenting speed of a suspension," Phys. Fluids 28, 759 (1985).

${ }^{4}$ E. J. Hinch, "Sedimentation of small particles," in Disorder and Mixing edited by E. Guyon, J.-P. Nadal, and Y. Pomeau (Kluwer Academic, Dordrecht, 1988), p. 153.

${ }^{5} \mathrm{H}$. Nicolai and E. Guazzelli, "Effect of the vessel size on the hydrodynamic diffusion of sedimenting spheres," Phys. Fluids 7, 3 (1995).

${ }^{6}$ P. N. Segrè, E. Helbolzheimer, and P. M. Chaikin, "Long-range correlations in sedimentation," Phys. Rev. Lett. 79, 2574 (1997).

${ }^{7}$ É. Guazzelli, "Evolution of particle-velocity correlations in sedimentation," Phys. Fluids 13, 1537 (2001).

${ }^{8}$ L. Bergougnoux, S. Ghicini, E. Guazzelli, and E. J. Hinch, "Spreading fronts and fluctuations in sedimentation," Phys. Fluids 15, 1875 (2003).

${ }^{9}$ J. H. C. Luke, "Decay of velocity fluctuations in a stably stratified suspension," Phys. Fluids 11, 754 (1999).

${ }^{10}$ S.-Y. Tee, P. J. Mucha, L. Cipelletti, S. Manley, M. P. Brenner, P. N. Segrè, and D. A. Weitz, "Nonuniversal velocity fluctuations of sedimenting particles," Phys. Rev. Lett. 89, 054501 (2002).

${ }^{11}$ P. J. Mucha, S.-Y. Tee, D. A. Weitz, B. I. Shraiman, and M. P. Brenner, "A model for velocity fluctuations in sedimentation," J. Fluid Mech. 501, 71 (2004). 